\title{
Analysis of the reproducibility of the gray values and noise of a direct digital radiography system
}

\section{Marcelo Lupion POLETI ${ }^{(a)}$ Thais Maria Freire FERNANDES(a) \\ Renata Cordeiro TEIXEIRA ${ }^{\text {(b) }}$ Ana Lúcia Alvares CAPELOZZA(c) Izabel Regina Fischer RUBIRA-BULLEN(c)}

(a) Universidade Norte do Paraná - UNOPAR, Faculty of Dentistry, Department of Orthodontics, Londrina, PR, Brazil.

(b) Universidade de Fortaleza - UNIFOR, Faculty of Dentistry, Department of Stomatology, Fortaleza, CE, Brazil.

(c) Universidade de São Paulo - USP, Bauru School of Dentistry, Department of Stomatology, Bauru, SP, Brazil.
Declaration of Interests: The authors certify that they have no commercial or associative interest that represents a conflict of interest in connection with the manuscript.

\section{Corresponding Author:}

Marcelo Lupion Poleti

E-mail:marcelo_poleti@yahoo.com.br

DOI: 10.1590/1807-3107BOR-2015.vol29.0062

Submitted: Out 09, 2014

Accepted for publication: Jan 21, 2015

Last revision: Apr 22, 2015

\begin{abstract}
The aim of this study was to evaluate the reproducibility of the gray values and noise of a direct digital radiography system (Visualix eHD) for various exposure times and analyzed regions. To obtain radiographic images in a standardized manner, the digital sensor of the system and a stepwedge were positioned in a phantom at a focus-film distance of $30 \mathrm{~cm}$ in a dental device at $70 \mathrm{kV}, 7 \mathrm{~mA}$ and $2.2 \mathrm{~mm}$ filtration. Ten consecutive repetitions of X-ray imaging were performed at each exposure time $(0.05,0.07,0.09$ and $0.13 \mathrm{~s})$. Gray values were analyzed using Image software in five regions of interest (ROIs): alveolar bone (AB), soft tissue (ST) and three steps of the stepwedge (S1, S2 and S3). The results showed that both the variability of the gray values and the noise were statistically greater $(p<0.05)$ in the most radiolucent region (ST). Only the noise was affected by the exposure time. In conclusion, the reproducibility of the gray values and the noise of the Visualix eHD system can vary in specific areas with different radiolucency.
\end{abstract}

Keywords: Radiography, Dental, Digital; Reproducibility of Results; Diagnostic Imaging.

\section{Introduction}

The technique of digital radiography is similar to that of conventional (analogic) radiology; however, a computer is substituted for the chemical processing phase. This substitution is advantageous because it eliminates technical errors inherent in chemical processing. Other advantages of digital systems include fast processing and the ability to use the same sensor multiple times. ${ }^{1,2,3,4,5,6}$ The image visualization programs used in digital radiography systems have various post-processing resources, enabling the user to alter brightness and contrast, rotate and amplify or reduce the image, invert the gray scale, apply pseudocolors to different gray tones, perform linear and angular measurements and quantify gray values in a region of interest (ROI). $5,6,7,8,9,10,11$

Damante et al..$^{10}$ developed a protocol for radiographic diagnosis and follow-up studies of simple bone cysts based on gray values. The authors evaluated ten patients and concluded that it is possible to apply a non-interventional follow-up protocol that avoids unnecessary surgery of these lesions. Ferreira Junior et al. ${ }^{11}$ also performed a study using gray values for the diagnosis of simple bone cysts and 
odontogenic keratocysts. However, gray values can vary, depending on the quality of the system used and on the exposure time. ${ }^{6,7,8}$ As a result, these situations can compromise the diagnosis and follow-up studies of the lesions.

Various studies have evaluated the physical and clinical outcomes associated with digital systems under different conditions. ${ }^{1,3,4,5,6,7,9,12,13,14,15,16,17}$ However, the current literature does not include studies of the reproducibility of gray values and noise associated with the Visualix eHD system when using gray values analysis to diagnose and track lesions. ${ }^{8,10,11,12}$ Therefore, the objective of this study was to evaluate the reproducibility of the gray values and noise associated with the Visualix eHD system when varying the exposure times and the regions analyzed.

\section{Methodology}

For this study, the charge-coupled device (CCD) sensor (universal size) of the Visualix eHD system (Gendex Dental Systems, Lake Zurich, USA) was used, which had dimensions of $37.5 \times 25.5 \mathrm{~mm}$, a pixel size of $19.5 \mu \mathrm{m}$ and a spatial resolution of $25.6 \mathrm{lp} / \mathrm{mm}$. The CCD sensor was positioned in a phantom with a soft tissue simulator (acrylic), such that radiographic imaging could be performed in a standardized manner, without altering the position of the images. The dental X-ray machine (Kaycor X-707, Yoshida Dental MFG Co. Ltd., Tokyo, Japan) was operated with $70 \mathrm{kV}, 7 \mathrm{~mA}, 2.2 \mathrm{~mm}$ filtration and a focus-film distance of $30 \mathrm{~cm}$. A stepwedge with three steps, corresponding to $0.2,0.4$ and $0.6 \mathrm{~mm}$ of thickness, was coupled to the sensor. Ten consecutive radiographic images were acquired without altering the position of the sensor for each exposure time selected $(0.05,0.07,0.09$ and $0.13 \mathrm{~s})$ (Figure 1).

Radiographic images were obtained, processed and stored in TIFF format (with no compression) and opened in the ImageJ program (National Institutes of Health, Washington, USA) on a computer with a 15-inch monitor (Satellite, Toshiba, Tokyo, Japan). For each image, five areas corresponding to ROIs were opened in the same square format $(102 \times 102$ pixels), including three steps of the stepwedge (S1, S2 and S3), one for soft tissue (ST) and one for alveolar bone (AB) (Figure 2). All of the areas were saved so that their sizes and formats would be maintained for all measurements. The mean gray values of the ROIs were determined using the Analyse/Measure tool of the ImageJ program. $5,6,7,8,10,11$

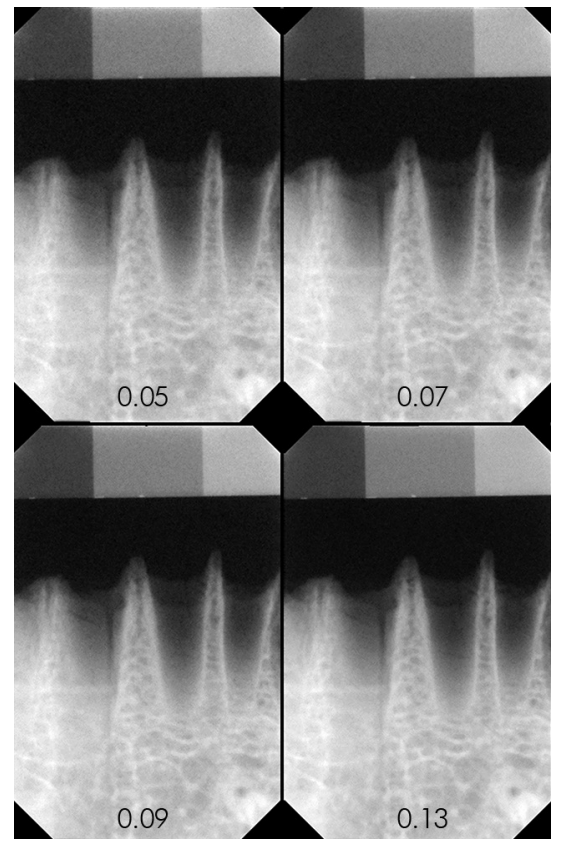

Figure 1. Images of the different groups.

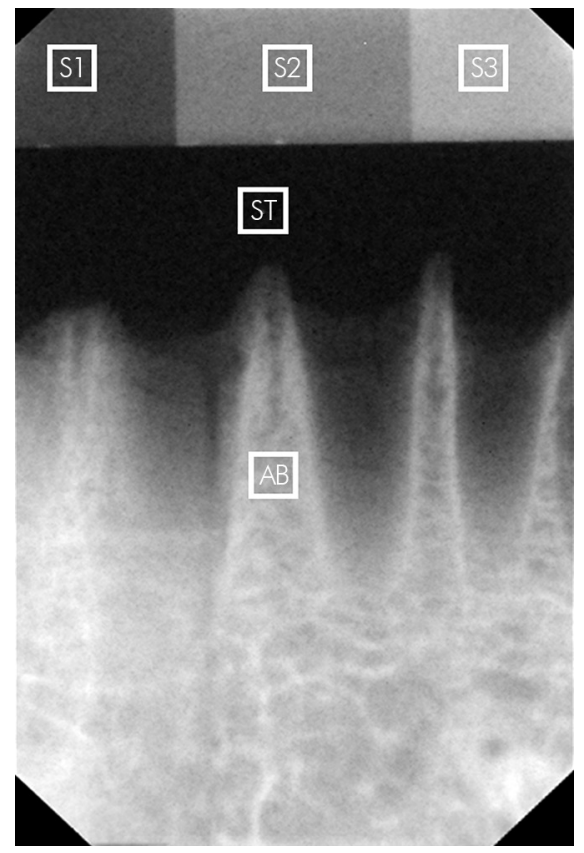

Figure 2. Definitions of the regions of interest (ROls). 
To analyze the reproducibility of the gray values, the following formula was used: (highest difference/mean) $\times 100$. The highest difference corresponds to the mean gray value minus the lowest gray value or the highest gray value minus the mean gray value in the ROI on each image. ${ }^{5}$ The mean, highest and lowest gray values were obtained after ten consecutive scannings of the sensor for each of the four exposure times and for each of the five regions. The noise was calculated in the ROI of the images. The formula applied to calculate the noise was: (standard deviation [SD]/gray value) $\times 100 .^{8}$ The means of the standard deviation and gray value for each ROI were obtained from ten consecutive radiographic images.

The reproducibility of the gray values and noise for the various exposure times and for each region analyzed were compared using analysis of variance (ANOVA). All statistical analyses were performed using Statistica for Windows 6.0 (Statistica for Windows, StatSoft Inc., Tulsa, USA). Statistically significant differences were defined as those with $\mathrm{p}<0.05$.

\section{Results}

Table 1 shows the results of comparisons of the gray values for various exposure times and ROIs. There were no significant differences between mean gray values evaluated for analyzed ROIs and exposure times $(\mathrm{p}>0.05)$.

There were no significant differences in the reproducibility of the gray values for each $\mathrm{ROI}$ at the different exposure times ( $p>0.05)$. However, there was a significant difference in the reproducibility between the soft tissue ROI and the other four ROIs $(p=0.000)$ at all four exposure times (Table 2).

Different letters in the vertical direction represent statistically significant differences (Tukey's test).

There were significant differences in the noise values between the different exposure times and ROIs ( $\mathrm{p}<0.05)$ (Table 3$)$.

Different uppercase letters in the horizontal direction represent statistically significant differences (Tukey's test).

Different lowercase letters in the vertical direction represent statistically significant differences (Tukey's test).

Table 1. Comparison of the gray values in the various exposure times and ROIs (ANOVA).

\begin{tabular}{|c|c|c|c|c|c|c|c|c|c|c|c|c|c|c|c|c|c|}
\hline \multirow{2}{*}{$\begin{array}{l}\text { Exposure } \\
\text { time }(s)\end{array}$} & \multicolumn{4}{|c|}{0.05} & \multicolumn{4}{|c|}{0.07} & \multicolumn{4}{|c|}{0.09} & \multicolumn{4}{|c|}{0.13} & \\
\hline & Minimum & Maximum & Mean & SD & Minimum & Maximum & Mean & SD & Minimum & Maximum & Mean & SD & Minimum & Maximum & Mean & SD & \\
\hline $\begin{array}{l}\text { Alveolar } \\
\text { bone }\end{array}$ & 191.58 & 193.90 & 192.96 & 0.68 & 192.30 & 194.24 & 193.30 & 0.63 & 192.67 & 194.08 & 193.42 & 0.47 & 192.66 & 194.29 & 193.21 & 0.53 & 0.695 \\
\hline $\begin{array}{l}\text { Soft } \\
\text { tissue }\end{array}$ & 21.72 & 24.26 & 22.63 & 0.84 & 20.82 & 23.43 & 21.88 & 0.85 & 19.58 & 22.89 & 21.26 & 1.05 & 18.07 & 20.31 & 18.69 & 0.68 & 0.658 \\
\hline S1 & 82.64 & 85.37 & 84.14 & 0.89 & 82.45 & 84.17 & 83.36 & 0.57 & 81.66 & 83.61 & 82.74 & 0.59 & 79.85 & 81.82 & 80.42 & 0.55 & 0.394 \\
\hline S2 & 148.06 & 150.25 & 149.10 & 0.67 & 147.39 & 149.75 & 148.83 & 0.76 & 147.64 & 149.31 & 148.74 & 0.56 & 146.55 & 148.42 & 147.19 & 0.46 & 0.508 \\
\hline S3 & 191.21 & 194.05 & 192.98 & 0.85 & 192.47 & 194.79 & 193.53 & 0.74 & 192.45 & 194.23 & 193.35 & 0.48 & 191.84 & 194.01 & 192.41 & 0.58 & 0.348 \\
\hline
\end{tabular}

Table 2. Comparison of the reproducibility of the gray values in the various exposure times and ROls.

\begin{tabular}{|c|c|c|c|c|c|c|c|c|c|}
\hline \multirow{2}{*}{ Exposure time $(\mathrm{s})$} & \multicolumn{2}{|c|}{0.05} & \multicolumn{2}{|c|}{0.07} & \multicolumn{2}{|c|}{0.09} & \multicolumn{2}{|c|}{0.13} & \multirow{2}{*}{$p$} \\
\hline & Mean & SD & Mean & SD & Mean & SD & Mean & SD & \\
\hline Alveolar bone & $0.35^{\circ}$ & 0.18 & $0.29^{a}$ & 0.18 & $0.20^{a}$ & 0.12 & $0.19^{a}$ & 0.09 & 0.309 \\
\hline Soft tissue & $3.23^{b}$ & 1.81 & $3.17^{b}$ & 2.22 & $4.09^{b}$ & 2.76 & $2.90^{\mathrm{b}}$ & 2.20 & 0.710 \\
\hline S1 & $0.92^{a}$ & 0.54 & $0.57^{\circ}$ & 0.38 & $0.59^{a}$ & 0.39 & $0.51^{a}$ & 0.46 & 0.223 \\
\hline S2 & $0.39^{a}$ & 0.23 & $0.44^{\circ}$ & 0.26 & $0.32^{a}$ & 0.19 & $0.20^{a}$ & 0.24 & 0.185 \\
\hline S3 & $0.36^{\circ}$ & 0.25 & $0.30^{\circ}$ & 0.24 & $0.20^{a}$ & 0.15 & $0.19^{a}$ & 0.23 & 0.309 \\
\hline$P$ & \multicolumn{2}{|c|}{0.000} & \multicolumn{2}{|c|}{0.000} & \multicolumn{2}{|c|}{0.000} & \multicolumn{2}{|c|}{0.000} & \\
\hline
\end{tabular}

SD: Standard deviation.

Different letters in the vertical direction represent statistically significant differences (Tukey's test). 
Table 3. Comparison of the noise in the various exposure times and ROls.

\begin{tabular}{|c|c|c|c|c|c|c|c|c|c|}
\hline \multirow{2}{*}{ Exposure time (s) } & \multicolumn{2}{|c|}{0.05} & \multicolumn{2}{|c|}{0.07} & \multicolumn{2}{|c|}{0.09} & \multicolumn{2}{|c|}{0.13} & \multirow{2}{*}{$p$} \\
\hline & Mean & SD & Mean & SD & Mean & SD & Mean & SD & \\
\hline Alveolar bone & $4.89^{\mathrm{A}, \mathrm{a}}$ & 0.11 & $4.95^{\mathrm{A}, \mathrm{a}}$ & 0.12 & $4.98^{\mathrm{A}, \mathrm{a}}$ & 0.09 & $5.17^{\mathrm{B}, \mathrm{a}}$ & 0.08 & 0.000 \\
\hline Soft tissue & $26.30^{A, B, b}$ & 1.12 & $25.53^{A, B, b}$ & 1.41 & $25.08^{A, b}$ & 1.02 & $26.88^{\mathrm{B}, \mathrm{b}}$ & 1.00 & 0.010 \\
\hline S1 & $6.25^{A, c}$ & 0.16 & $5.84^{\mathrm{B}, \mathrm{c}}$ & 0.17 & $5.61^{c, c}$ & 0.16 & $5.18^{\mathrm{D}, \mathrm{a}}$ & 0.11 & 0.000 \\
\hline S2 & $3.09^{\mathrm{A}, \mathrm{d}}$ & 0.09 & $2.82^{\mathrm{B}, \mathrm{d}}$ & 0.07 & $2.60^{c, d}$ & 0.09 & $2.33^{\mathrm{D}, \mathrm{c}}$ & 0.11 & 0.000 \\
\hline S3 & $2.13^{\mathrm{A}, \mathrm{e}}$ & 0.06 & $1.91^{\mathrm{B}, \mathrm{e}}$ & 0.09 & $1.75^{\mathrm{C}, \mathrm{e}}$ & 0.04 & $1.50^{\mathrm{D}, \mathrm{d}}$ & 0.07 & 0.000 \\
\hline$p$ & \multicolumn{2}{|c|}{0.000} & \multicolumn{2}{|c|}{0.000} & \multicolumn{2}{|c|}{0.000} & \multicolumn{2}{|c|}{0.000} & \\
\hline
\end{tabular}

SD: Standard deviation.

Different uppercase letters in the horizontal direction represent statistically significant differences (Tukey's test).

Different lowercase letters in the vertical direction represent statistically significant differences (Tukey's test).

\section{Discussion}

No studies to date have physically analyzed the reproducibility of the gray values and noise associated with the Visualix eHD system. The results of this study demonstrate the reproducibility of the gray values and noise for the system in five regions of radiographic imaging when the exposure time was varied.

Our study did not detect a statistically significant difference between the gray values with different exposure times, perhaps because there was relatively little variation in the exposure times selected (ranging from 0.05 to 0.13 ) or because the phantom with the soft tissue simulator attenuated the radiation. ${ }^{5}$ According to Pietrobelli et al. ${ }^{18}$ attenuation occurs when the photon signal crosses an element, chemical component or solution, spontaneously reducing the intensity through atomic interactions and leading to the absorption and dissipation of photons. Thus, when an X-ray beam penetrates an analyzed region, the presence of soft and hard tissues in this trajectory influences the gray values. In this study, the highest gray values were observed in the most radiopaque regions ( $\mathrm{AB}$ and S3), and the lowest values were observed in the most radiolucent regions (ST and S1) $5,8,16,17$ (Table 1).

No significant differences were found between the reproducibility of the gray values $(p \geq 0.185)$ for various exposure times $(0.05,0.07,0.09$ and 0.13 $\mathrm{s})$, because the gray values were not influenced by the exposure times. However, the reproducibility of the gray values did vary between the soft tissue ROI and the other four ROIs $(p=0.000)$. This result demonstrated that the variability of the gray shades after consecutive repetitions of the same ROI was greater in more radiolucent ROIs (Table 2). The higher mean variability of the gray shades for the Visualix eHD system in this study was $4.09 \%$, which is lower than the results reported for the Digora system, i.e., $75 \%$ by Freitas et al. ${ }^{5}$ and $27.95 \%$ by Teixeira et al. ${ }^{17}$

The lack of reproducibility of gray values of radiographic systems could be deleterious when limitations are unknown, because the system could potentially attribute differences in gray values as corresponding to a true change in the clinical condition of a patient. This situation could compromise the diagnosis and follow-up studies when a quantitative analysis of gray values is used, ${ }^{5}$ with clinical implications. For example, when a carious lesion is relatively large, the area analyzed will be more radiolucent, and there will be greater variation in gray values. In contrast, when gray values analysis is used to evaluate the evolution of bone lesions, ${ }^{10,11}$ the area analyzed will be more radiopaque, leading to lower variability of the gray values and more accurate analysis using the Visualix eHD system. This finding is supported by the studies of Damante et al..$^{10}$ and Ferreira Junior et al. ${ }^{11}$ which proposed a protocol for the radiographic diagnosis and follow-up of simple bone cysts and odontogenic keratocysts based on gray values of the images.

A key factor for successful radiographic imaging is that the X-ray signal detected by the sensor must overcome the inherent noise of the digital radiographic system. ${ }^{6}$ Noise is the undesirable fluctuation of pixel intensities, which can compromise the image studied, ${ }^{12,17}$ thereby affecting the grey-scale intensity and, consequently, the gray values. ${ }^{6,7,17}$ Noise levels can vary, depending on the quality of the system used ${ }^{6,7,8}$ and the exposure time. ${ }^{6}$ Moreover, noise levels can be evaluated using various physical parameters, such as the standard deviation. ${ }^{6,7}$ 
In this study, we identified differences in noise levels for various exposure times and ROIs. The noise decreased with increasing exposure times for the three areas of the stepwedge (Table 3). This finding differs from a study by Wenzel, ${ }^{6}$ which found an increase in noise with an increase in exposure time from 0.20 to $0.60 \mathrm{~s}$. The reason for this difference is based on the dose of radiation needed to saturate the sensor used in each of these studies. ${ }^{6}$ In our study, the exposure times were smaller (ranging from 0.05 to $0.13 \mathrm{~s}$ ), whereas the study by Wenzel's had longer exposure times (ranging from 0.20 to $0.60 \mathrm{~s}$ ). On the other hand, our study found that the noise was statistically significantly greater when the area analyzed was more radiolucent (i.e., soft tissue). These findings are in accordance with the results reported by Rubira-Bullen et al. ${ }^{8}$ for digital radiographic images. Thus, as observed in the analysis of the reproducibility

\section{References}

1. Grondahl HG. Digital radiology in dental diagnosis: a critical view. Dentomaxillofac Radiol. 1992 Nov;21(4):198-202.

2. Vandre RH, Webber RL. Future trends in dental radiology. Oral Surg Oral Med Oral Pathol Oral Radiol Endod. 1995Oct;80(4):471-8.

3. Versteeg CH, Sanderink GC, van der Stelt PF. Efficacy of digital intra-oral radiography in clinical dentistry. J Dent. 1997 May-Jul;25(3-4):215-24.

4. Pascon EA, Marrelli M, Congi O, Ciancio R, Miceli F, Versiani MA. An in vivo comparison of working length determination of two frequency-based electronic apex locators. Int Endod J. 2009 Nov;42(11):1026-31.

5. Freitas P, Yaedu RY, Rubira-Bullen IR, Escarpinati M, Vieira MC, Schiabel H, et al. Reproducibility of pixel values for two photostimulable phosphor plates in consecutive standardized scannings. Braz Oral Res. 2006 Jul-Sep;20(3):207-13.

6. Wenzel A. Sensor noise in direct digital imaging (the RadioVisioGraphy, Sens-a-Ray, and Visualix/Vixa systems) evaluated by subtraction radiography. Oral Surg Oral Med Oral Pathol. 1994 Jan;77(1):70-4.

7. Haiter-Neto F, Wenzel A. Noise in subtraction images made from pairs of bitewing radiographs: a comparison between two subtraction programs. Dentomaxillofac Radiol. 2005 Nov;34(6):357-61.

8. Rubira-Bullen IR, Escarpinati MC, Schiabel H, Vieira MA, Rubira $\mathrm{CM}$, Lauris JR. Evaluating noise in digitized radiographic images by means of histogram. J Appl Oral Sci. 2006 Dec;14(6):410-4.

9. Nelvig P, Wing K, Welander U. Sens-A-Ray. A new system for direct digital intraoral radiography. Oral Surg Oral Med Oral Pathol. 1992 Dec;74(6):818-23. of the gray values in this study, as the noise of the system used increases, the interference for the quantitative analysis of the gray values also increases, especially in radiolucent lesions, such as dental caries.

This study did not assess the stability of the output power of the dental X-ray machine used. However, any instability of the machine was not sufficient to alter the reproducibility of the gray values or the noise.

Studies that evaluate the physical analysis of the radiographic systems are important to show the limitations and improve the diagnosis of lesions and follow-up studies.

\section{Conclusions}

Based on the methods used, the reproducibility of the gray values and noise of the Visualix eHD system can vary in specific areas with different radiolucency.

10. Damante JH, Guerra ENS, Ferreira Junior O. Spontaneous resolution of simple bone cysts. Dentomaxillofac Radiol. 2002 May;31(3):182-6.

11. Ferreira Junior O, Damante JH, Lauris JR. Simple bone cyst versus odontogenic keratocyst: differential diagnosis by digitized panoramic radiography. Dentomaxillofac Radiol. 2004 Nov;33(6):373-8.

12. Attaelmanan AG, Borg E, Grondahl HG. Signal-to-noise ratios of 6 intraoral digital sensors. Oral Surg Oral Med Oral Pathol Oral Radiol Endod. 2001 May;91(5):611-5.

13. Borg E, Attaelmanan A, Grondahl HG. Subjective image quality of solid-state and photostimulable phosphor systems for digital intra-oral radiography. Dentomaxillofac Radiol. 2000 Mar;29(2):70-5.

14. Hayakawa Y, Farman AG, Kelly MS, Kuroyanagi K. Intraoral radiographic storage phosphor image mean pixel values and signal-to-noise ratio: effects of calibration. Oral Surg Oral Med Oral Pathol Oral Radiol Endod. 1998 Nov;86(5):601-5.

15. Molteni R. Direct digital dental x-ray imaging with visualix/ VIXA. Oral Surg Oral Med Oral Pathol. 1993 Aug;76(2):235-43.

16. Rasimick BJ, Shah RP, Musikant BL, Deutsch AS. Radiopacity of endodontic materials on film and a digital sensor. J Endod. 2007 Sep;33(9):1098-101.

17. Teixeira RC, Rubira-Bullen IRF, Rubira CMF, Lauris JR. Avaliação da reprodutibilidade do valor de pixel no sistema Digora. Rev ABRO. 2007;8(2):86-93.

18. Pietrobelli A, Formica C, Wang Z, Heymsfield SB. Dual-energy X-ray absorptiometry body composition model: review of physical concepts. Am J Physiol. 1996 Dec;271(6 Pt 1):E941-51. 